\title{
Segmentação Automática da Próstata em Imagens de Ressonância Magnética utilizando Redes Neurais Convolucionais e Mapa Probabilístico
}

\author{
Jonnison L. Ferreira ${ }^{1}$, Giovanni L. F. da Silva ${ }^{1}$, Artur B. S. Reis ${ }^{1}$, \\ André B. Cavalcante ${ }^{1}$, Aristófanes C. Silva ${ }^{1}$, Anselmo C. de Paiva ${ }^{1}$ \\ ${ }^{1}$ Núcleo de Computação Aplicada - Universidade Federal do Maranhão (UFMA) \\ CEP 65080-805 - São Luís - MA - Brasil \\ \{jonnison, giovannilucca, arturberna, ari, paiva\}@nca.ufma.br, \\ abcborges lgmail.com
}

\begin{abstract}
Prostate cancer is the second most common type of cancer among men, and prostate cancer screening has been increasing for prevention, diagnosis and treatment. Manual segmentation of the prostate is extremely timeconsuming and prone to variability among different specialists, suggesting the development of automatic techniques for prostate segmentation. In this work, we propose a fully automatic method for the prostate segmentation from magnetic resonance imaging using a deep learning technique and probabilistic map. The experimental results obtained here indicate a satisfactory segmentation, considering that we obtain an average Dice similarity coefficient of $85.17 \%$.
\end{abstract}

Resumo. O câncer de próstata é o segundo tipo de câncer mais comum entre os homens e atualmente tem crescido a utilização de exames de imagens da próstata para a prevenção, diagnóstico e tratamento. A segmentação manual da próstata é extremamente demorada e propensa à variabilidade entre diferentes especialistas, o que sugere o desenvolvimento de técnicas automáticas para a segmentação da próstata. Neste trabalho, propomos um método totalmente automático para a segmentação da próstata a partir de imagens de ressonância magnética usando uma técnica de aprendizado profundo e mapa probabilístico. Os resultados experimentais aqui obtidos indicam uma segmentação satisfatória, tendo em vista que obtemos um coeficiente de similaridade de Dice médio de $85,17 \%$.

\section{Introdução}

O câncer de próstata, de acordo com o Instituto Nacional do Câncer (INCA), é o segundo tipo de câncer mais comum entre os homens (atrás apenas do câncer de pele nãomelanoma), e considerando ambos os sexos é o quarto tipo mais comum [INCA 2018]. A American Cancer Society estima que um a cada sete homens no mundo, sejam diagnosticados com câncer de próstata durante sua vida [Society 2018]. No Brasil, estima-se em 2018, cerca de 68.220 novos casos diagnosticados [INCA 2018].

As duas maneiras mais comuns para a detecção dessa patologia são o exame do antígeno prostático específico (PSA) no sangue e o exame palpatório (DRE). Esses dois tipos de exames dependem que a anatomia da próstata esteja determinada corretamente. No entanto, a segmentação manual da próstata é extremamente demorada e 
propensa à variabilidade entre diferentes especialistas, o que sugere o desenvolvimento de técnicas para a segmentação automática da próstata baseadas em imagens digitais [Mahapatra and Buhmann 2014]. Uma boa metodologia de segmentação automática da próstata deve superar alguns desafios, tais como a variação do tamanho e formato da próstata e as diferenças entre os protocolos de aquisição das imagens.

Entre as modalidades de exames por imagem, a ressonância magnética (RM) se mostra o método mais preciso disponível para obter informações sobre o tamanho, forma e localização da próstata, devido sua capacidade de gerar uma alta resolução espacial e contraste em tecidos moles.

Portanto, este artigo tem como objetivo apresentar um método totalmente automático para a segmentação da próstata a partir de imagens de RM, usando a técnica Redes Neurais Convolucionais (CNN) e um mapa probabilístico da próstata. A principal contribuição desse trabalho é o uso da informação do mapa probabilístico da próstata, visto que as demais abordagens utilizam apenas informações da imagem de RM. No modelo proposto, o mapa probabilístico é utilizado como uma informação a priori da localização e forma da próstata, contribuindo na tarefa de aprendizado da rede.

Este artigo está organizado da seguinte forma: na Seção 2 são apresentados os trabalhos relacionados com o tema da pesquisa. A descrição do método proposto é detalhada na Seção 3. Os experimentos e resultados para validar a pesquisa e um estudo comparativo com outros trabalhos equivalentes são relatados na Seção 4. Finalmente, as conclusões e trabalhos futuros são abordados na Seção 5.

\section{Trabalhos Relacionados}

Na literatura estão disponíveis trabalhos relacionados com o problema de segmentação da próstata utilizando imagens de ressonância magnética. A seguir são apresentados alguns desses trabalhos.

As abordagens baseadas em forma e contorno são bastantes utilizadas na segmentação do volume da próstata. Em [Maan and van der Heijden 2012] são utilizados registro não rígido, modelos de aparência ativos (AAM) e análise de componentes principais (PCA). Inicialmente é feito o registro, em seguida são construídos os AAMs. A última etapa utiliza a PCA para determinar as principais variações de formato da próstata. Esta abordagem obteve um valor médio de $81 \%$ para o coeficiente de similaridade de Dice (DSC).

No trabalho de [Al-Qunaieer et al. 2014] é proposto a utilização de Level Sets em multiresolução. Esta estratégia de multiresolução é aplicada para acelerar as Level Sets. Este método é usado em cada fatia dos exames e as configurações de resoluções são semiautomáticas. Neste trabalho apresentou um valor médio de 79\% para o DSC.

Em [Li et al. 2013] é proposta uma segmentação utilizando mapa probabilístico e caminhos aleatórios. Inicialmente, é gerado o mapa probabilístico da próstata calculando a probabilidade de ocorrência dos voxels na próstata, o volume segmentado é obtido através do caminho aleatório. Este método obteve um valor médio do DSC de 80,7\%.

O trabalho de [Zhu et al. 2017] propôs uma arquitetura de uma rede totalmente convolucional semelhante a U-Net, porém é realizado um troca de informações residuais em cada camada de convolução, adicionando uma camada residual a cada estágio 
da arquitetura U-Net. Para avaliar a metodologia, é utilizada uma base privada para segmentação da próstata, composta por 81 exames, sendo usado quatro para teste e os demais para treino, este trabalho obteve DSC médio de $88 \%$.

A PSNet proposta em [Tian et al. 2018] apresenta uma rede totalmente convolucional (FCN), modificando apenas as três últimas camadas do modelo original da FCN para adequação da base. O modelo foi testado em três bases contendo num total de 140 exames de RM de próstata, obtendo um DSC médio de $85 \%$.

O trabalho de [Karimi et al. 2017] define um método para segmentação automática de próstata que utiliza uma CNN global, que determinará uma bounding box da próstata, a qual é reamostrada e enviada para uma CNN local para uma delineação precisa do limite da próstata. Dessa forma, a CNN local pode efetivamente aprender a segmentar os detalhes sutis, que distinguem a próstata do tecido circundante usando a pequena quantidade de dados de treinamento disponíveis. Esse método foi testado na base PROMISE12 e o seu conjunto de validação obteve como resultado DSC de 84,9\% para rede global, 90,4\% para rede local e 91,2\% após o refinamento.

Por fim, em [Yang et al. 2016] é proposto um método semi-automático para segmentação baseado em contorno ativo Level Set. O método é dividido em três fases: na primeira fase, a fatia média dos dados da próstata é segmentada artificialmente para oferecer a informação prévia; na segunda fase, a detecção da próstata é implementada pela estimativa de similaridade da apresentação da textura; e na última fase, é aplicado um procedimento de restrição de textura para evitar a delineação do contorno falso. Esse método foi aplicado em uma base com 72 imagens de 18 indivíduos diferentes e obteve um DSC médio de $91 \%$.

Estes são exemplos de metodologias que foram desenvolvidas para segmentação de próstata em exames de RM. Dois pontos importantes comuns nestes trabalhos são a necessidade de uma fase de refinamento após a segmentação inicial e o uso de uma grande base para treino do método. Em nosso método tentar-se-á explorar estas deficiências com o intuito de melhorar as métricas que avaliam o desempenho em um número maior de casos.

\section{Materiais e Método}

Nesta seção é apresentado o método proposto para segmentação da próstata, a partir de imagens de ressonância magnética da próstata. Para isso, analisamos a capacidade da CNN baseada na arquitetura U-Net aplicada a segmentação de imagens. A Figura 1 apresenta as etapas deste método, juntamente com as técnicas utilizadas em cada etapa, que estão divididas em três partes: materiais, pré-processamento e segmentação.

\subsection{Materiais}

A base de imagens utilizada neste trabalho é composta por um subconjunto de 30 exames, disponibilizados juntamente com a marcação do especialista, no desafio de segmentação automatizada de estruturas da próstata NCI-ISBI em 2013 [Bloch et al. 2015]. O desafio foi organizado pelo National Cancer Imaging Archive (NCIA) e está disponível em The Cancer Imaging Archive (TCIA) [Bloch et al. 2015].

As imagens foram obtidas de exames de RM com ponderação em T2, campo de $3 \mathrm{~T}$ e plano de aquisição axial. As imagens possuem 16 bits por voxel, espaçamento 


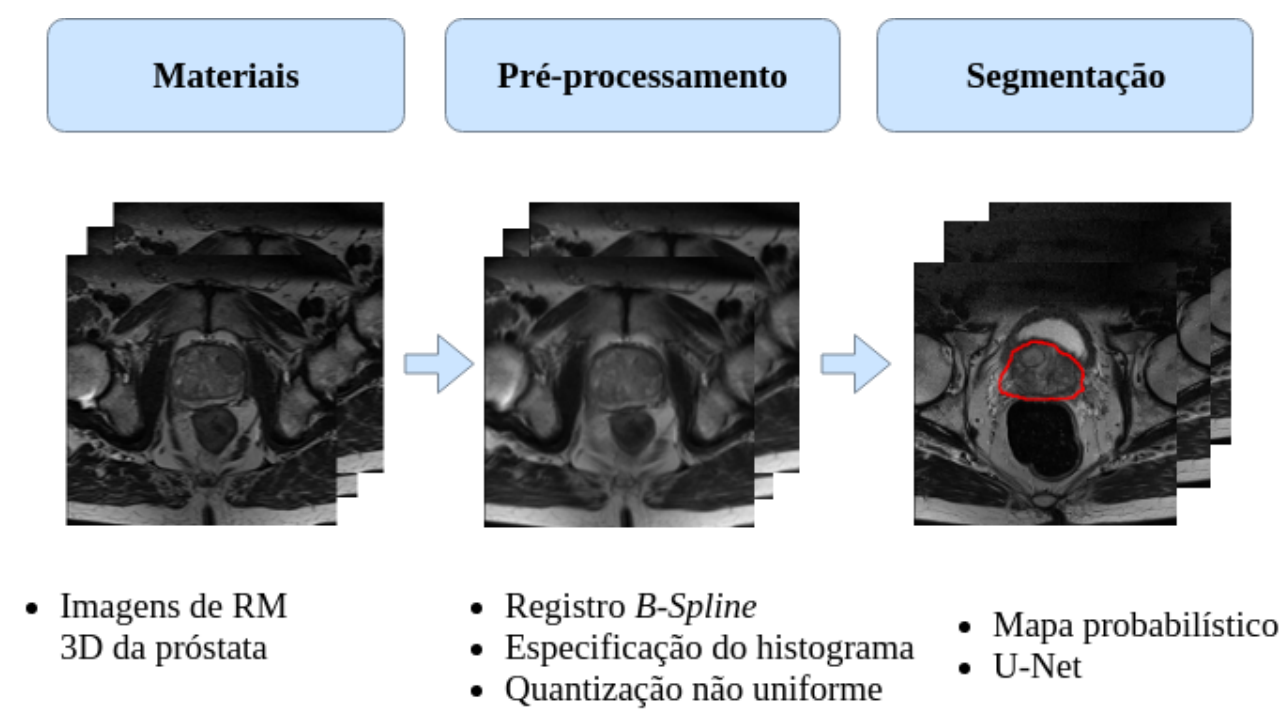

Figura 1. Etapas do método proposto.

entre voxels de $0.6 \mathrm{~mm} \times 0.6 \mathrm{~mm}$ e a espessura em cada fatia é de $4 \mathrm{~mm}$. Os exames possuem resolução de 320 x 320 pixels com o número de fatias variando de 15 a 24 [Smith et al. 2015].

\subsection{Pré-processamento}

A preparação das imagens para segmentação ocorre em três etapas de pré-processamento. Na primeira etapa é feito o registro das imagens, uma vez que as imagens da base não possuem os mesmos números de fatias e origem de sistema de coordenadas. Portanto, utilizou-se o algoritmo $B$-Splines para fazer o registro não rígido das imagens de RM. A escolha do $B$-Splines como método de transformação se deve ao fato da translação de um único ponto depender apenas da vizinhança imediata a ele [Yang et al. 2014]. Isto resulta em uma transformação controlada localmente e por consequência, menor deformidade nas imagens registradas.

$\mathrm{Na}$ segunda etapa é realizada a especificação do histograma. Em geral, a especificação do histograma é o processo de transformar o histograma de uma imagem origem para corresponder ao histograma de uma determinada imagem de modelo, para que ambas tenham uma distribuição de pixels semelhante [Gonzalez and Woods 2008]. Esse processo é realizado para que as imagens da base se tornem mais uniformes, já que no processo de aquisição, elas são obtidas por meio de protocolos e equipamentos diferentes. Então o histograma do primeiro exame é selecionado para especificação do histograma dos demais pacientes.

Por fim, a terceira e última etapa é aplicado a quantização não-uniforme da imagem para normalização dos valores de voxels entre 0 e 255. A Figura 2 apresenta o resultado de cada etapa do pré-processamento. 


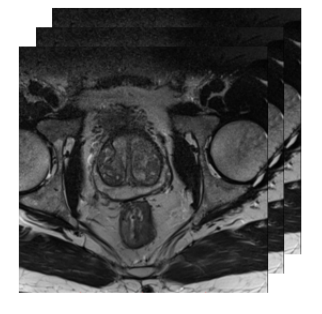

Imagem Original

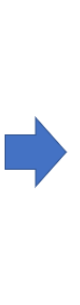

Imagem Registrada

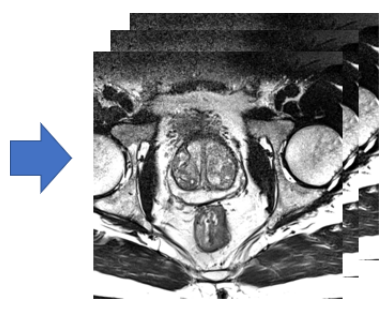

Imagem Especificada

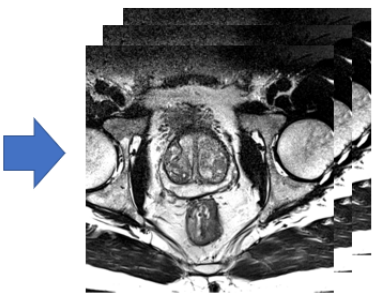

Imagem Quantizada

Figura 2. Etapas do pré-processamento das imagens.

\subsection{Segmentação}

\subsubsection{Mapa Probabilístico}

O mapa probabilístico representa a distribuição espacial dos voxels pertencentes ao volume da próstata nas marcações do especialista, onde cada elemento representa a probabilidade amostral do voxel pertencer a região da próstata. Para a construção do modelo denotado por $M$, consideramos $F_{i}(x, y, z)$ o voxel da posição $(x, y, z)$ do i-ésimo volume da base de treinamento que pertence ao objeto (próstata), definimos $p(F(x, y, z))$ como a probabilidade amostral do voxel da posição $(x, y, z)$ fazer parte do objeto, que é dada pela Equação 1

$$
p(F(x, y, z))=\frac{\sum_{i=0}^{n} F_{i}(x, y, z)}{n}
$$

onde $n$ é a quantidade de volumes da base de treinamento.

A Figura 3 demonstra a criação do mapa probabilístico baseado no conjunto de exames de treinamento, onde as tonalidades em vermelho indicam maior probabilidade do voxel pertencer a próstata enquanto as tonalidades mais próximas do azul indicam menores probabilidades.

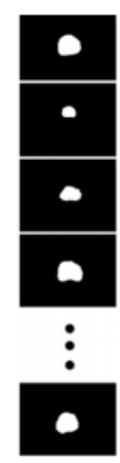

Marcação dos especialistas para uma determinada fatia dos exames de treinamento

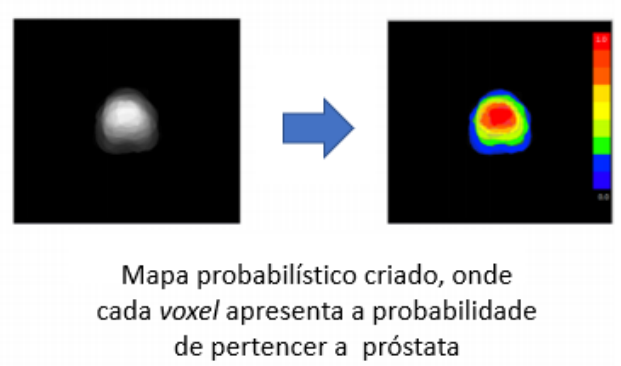

de pertencer a próstata

Figura 3. Criação do mapa probabilístico. 


\subsubsection{Rede Neural Convolucional}

O método de segmentação proposto é realizado por meio de uma rede neural convolucional (CNN). A CNN é uma arquitetura de aprendizado profundo composta por uma ou várias camadas convolucionais e subsequentes, seguidas opcionalmente de camadas totalmente conectadas, como em uma rede neural multicamada padrão [LeCun et al. 1998].

As CNNs possuem várias arquiteturas, seja para classificação, como a GoogLeNet [Szegedy et al. 2015], detecção, como VGG Net [Simonyan and Zisserman 2014], reconhecimento como AlexNet [Krizhevsky et al. 2012], dentre outras. Para a segmentação da próstata, foi utilizado a arquitetura de CNN U-Net [Ronneberger et al. 2015], que é um modelo proposto para segmentação de imagens biomédicas que tem como principal característica a utilização de poucas imagens no treinamento.

A U-Net é uma rede totalmente convolucional, cuja arquitetura consiste em um caminho de contratação para capturar o contexto, e um caminho de expansão simétrico que permite uma segmentação precisa. A principal estratégia que diferencia a U-Net das outras arquiteturas FCN é a combinação entre os mapas de características do estágio de contração e seus correspondentes simétricos no estágio de expansão, permitindo a propagação de informações de contexto para os mapas de características de alta resolução. Esse modelo vem superando os métodos existentes em diversos desafios biomédicos de segmentação de imagens [Ronneberger et al. 2015]. A Figura 4 ilustra a arquitetura e as camadas que constituem a rede.

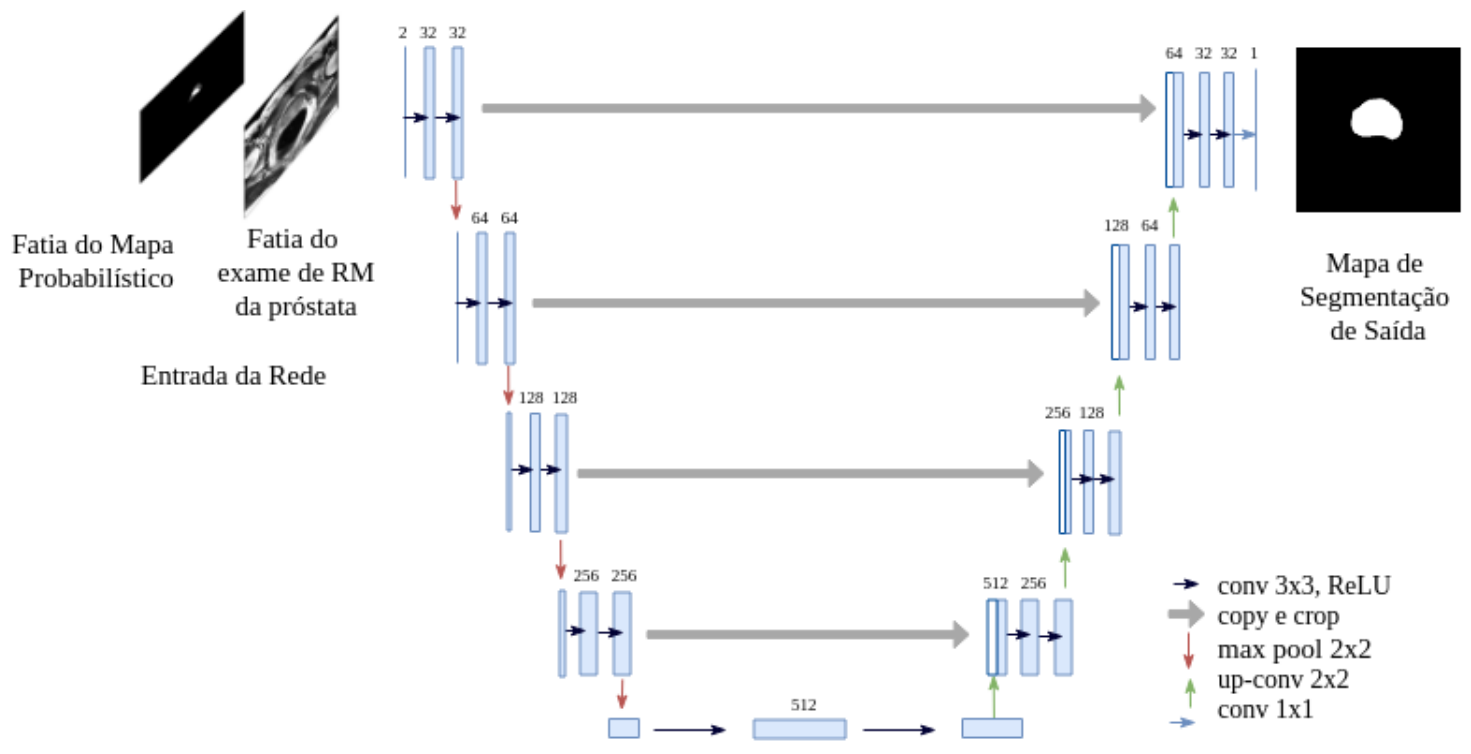

Figura 4. Representação da Arquitetura U-Net.

A distância entre as fatias de imagens de RM nos eixos x e y é maior, e as características tridimensionais podem ser imprecisos devido a resolução no eixo z. Assim, cada fatia bidimensional é servida como amostra de treinamento. Como ilustrado na Figura 4, a entrada da rede é uma imagem de dois canais composta pela concatenação da fatia do exame de RM pré-processada e da fatia do mapa probabilístico criado na Seção 3.3.1.

Na Figura 4, cada caixa azul corresponde a um mapa de características multi- 
canal. As setas indicam as operações realizadas a cada momento; setas azuis indicam convoluções $3 \times 3$, a quantidade de filtros está especificada em cima da caixa azul seguinte a seta; as setas cinzas indicam operação de cópia; as setas vermelhas indicam a operação de max pooling 2x2; as setas verdes operação de upsampling 2x2; por fim, na camada final é realizada uma operação de convolução 1x1 com uma função de ativação softmax para realizar a classificação pixel-a-pixel da imagem de saída, essa arquitetura e quantidades de filtros foram utilizados da maneira como foram definidas no trabalho de [Ronneberger et al. 2015].

\section{Resultados e Discussão}

Para avaliar o desempenho do método proposto são utilizadas as métricas de sensibilidade, especificidade, acurácia e área sob a curva Receiver Operating Characteristic (AUC) que são estatísticas comumente aplicadas na análise de imagens médicas [Duda 1973]. Além dessas métricas, nós utilizamos o coeficiente de similaridade Dice (DSC) que é a métrica padrão para avaliar a segmentação de imagens. O DSC é calculado pela Equação 2:

$$
\frac{2|A \cap B|}{|A|+|B|}
$$

onde $A$ é o mapa de segmentação de saída da rede e $B$ é a marcação do especialista.

Foram submetidas ao método todas as imagens da base, essas passaram primeiramente pela etapa de pré-processamento. As imagens dos 30 pacientes da base foram divididas aleatoriamente em três partes: 20 pacientes para treino, 5 para validação e 5 para teste. O treinamento foi realizado utilizando a arquitetura mostrada na Seção 3.3. As bases de treino e validação foram aumentadas por meio da aplicação de operações geométricas de rotação e espelhamento em cada fatia do exame, um total de sete operações em cada exame ( 3 rotações nos ângulos $90^{\circ}, 180^{\circ}$ e $270^{\circ}$ e 4 espelhamentos), como todos os exames são compostos por quinze fatias após a etapa de pré-processamento, a base de treino passou a conter 2.400 fatias e a base de validação 600 fatias. Para computar o gradiente descendente, utilizamos a função de perca entropia cruzada, juntamente com o método de otimização Adadelta [Zeiler 2012] para 200 épocas com taxa de aprendizagem 1 .

A Tabela 1 apresenta o desempenho do método proposto apresentando as médias das métricas obtidas nos volumes do conjunto de teste.

Tabela 1. Resultados obtidos no método proposto.

\begin{tabular}{ccccc}
\hline DSC & Sensibilidade & Especificidade & Acurácia & AUC \\
\hline $85,17 \%$ & $95,38 \%$ & $99,46 \%$ & $99,24 \%$ & $97,42 \%$ \\
\hline
\end{tabular}

Analisando a Tabela 1, observa-se que o método obteve resultados significativos, apresentando 85,17\% de DSC, esse valor indica que o volume segmentado obteve grande sobreposição em relação volume da próstata marcado pelo especialista, uma sensibilidade média de $95,38 \%$ e uma especificidade média de $99,46 \%$, demonstrando que o método consegue diferenciar os voxels pertencente a região da próstata ou fora da região. Baseado nos resultados, podemos verificar que há uma grande diferença entre o valor do DSC e as demais métricas, acredita-se que isso ocorra com as imagens em que as regiões próximas 
as bordas da próstata não estejam bem definidas, dificultando assim uma segmentação precisa.

A Figura 5 mostra algumas segmentações realizadas pelo método proposto, onde o contorno verde representa a marcação feita pelo especialista e o contorno vermelho representa a saída obtida pelo método proposto. A Figura 5 (A) mostra um caso em que a rede conseguiu segmentar corretamente a região da próstata. A Figura 5 (B) mostra uma segmentação considerada correta da próstata, porém com alguns falsos positivo e falsos negativo. A Figura 5 (C) mostra uma imagem onde a rede realiza a segmentação com falsos positivos, na fatia mostrada não há marcação do especialista e rede segmenta uma região falsa como sendo a próstata. Por fim, a Figura 5 (D) mostra uma imagem onde na fatia há uma região marcada pelo especialista e a rede não consegue segmentar a próstata. Esses erros ocorrem devido a textura semelhante entre a região de próstata e demais tecidos nas fatias iniciais de cada volume.
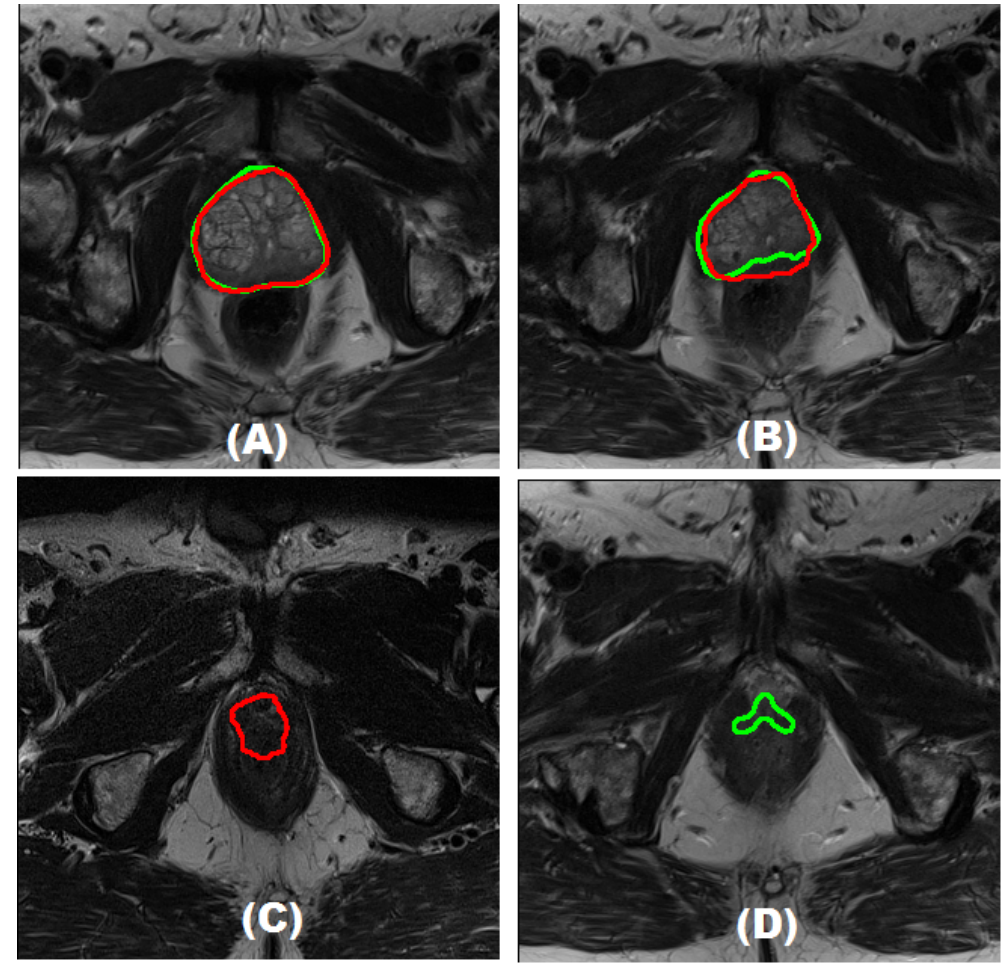

Figura 5. Resultados da Segmentação.

A Tabela 2 apresenta uma comparação dos resultados encontrados neste trabalho com alguns trabalhos relacionados. São apresentadas a base utilizada e as métricas usadas para validação. Este trabalho apresenta um melhor resultado em relação ao trabalho [Li et al. 2013] que utiliza a mesma base, mostrando resultado superior aos trabalhos de [Maan and van der Heijden 2012], [Al-Qunaieer et al. 2014] e [Tian et al. 2018] que utilizam outras bases. Nosso método apresenta um resultado inferior ao trabalho de [Yang et al. 2014], que por sua vez utiliza uma base privada com um método semiautomático, que depende de uma segmentação inicial feita por especialista. O trabalho de [Zhu et al. 2017] apresenta um resultado superior, porém utiliza uma base privada e um proporção muito pequena de exames para teste, a base possui 81 exames e apenas 4 são utilizados para testar a metodologia. O trabalho de [Karimi et al. 2017] também apre- 
senta resultado superior, mas é aplicada em uma base diferente da utilizada neste trabalho, necessitando de três níveis de segmentação.

Tabela 2. Comparação com métodos utilizados na literatura.

\begin{tabular}{|c|c|c|c|c|c|c|}
\hline Modelo & Base & DSC & Sensibilidade & Especifidade & Acurácia & AUC \\
\hline [Maan and van der Heijden 2012] & PROMISE12 & $81 \%$ & - & - & - & - \\
\hline [Li et al. 2013] & Prostate $3 \mathrm{~T}$ & $80,7 \%$ & - & - & - & - \\
\hline [Al-Qunaieer et al. 2014] & Prostate MR Image Database & $79 \%$ & - & - & - & - \\
\hline [Yang et al. 2016] & Privada & $91 \%$ & - & - & - & - \\
\hline [Zhu et al. 2017] & Privada & $88 \%$ & - & - & - & - \\
\hline [Karimi et al. 2017] & PROMISE12 & $91,12 \%$ & - & - & - & - \\
\hline [Tian et al. 2018] & ISBI2013 36 e PROMISE12 & $85 \%$ & - & - & - & - \\
\hline Método Proposto & Prostate 3T & $85,17 \%$ & $95,38 \%$ & $99,46 \%$ & $99,24 \%$ & $97,42 \%$ \\
\hline
\end{tabular}

\section{Conclusão}

No presente trabalho foi desenvolvido um método para a segmentação automática da próstata em imagens de RM. O método é dividido nas seguintes etapas: materiais, préprocessamento e segmentação. Os resultados obtidos neste trabalho demonstram um bom desempenho, obtendo valores médios de $85,17 \%$ para DSC, 95,38\% para sensibilidade, 99,46\% para especificidade, 99,25\% para acurácia e 97,42\% para AUC.

Observamos que para melhorar o desempenho geral da nossa metodologia devemos melhorar o DSC da segmentação, portanto, uma etapa de refinamento do contorno após a etapa de segmentação seria importante nesse aspecto.

A metodologia mostrou ser uma ferramenta útil para médicos especialistas na segmentação da próstata. Os resultados demonstraram um desempenho promissor utilizando a rede neural CNN e o mapa probabilístico para segmentação da próstata em imagens de ressonância magnética.

\section{Agradecimentos}

Os autores agradecem a Fundação de Amparo à Pesquisa e ao Desenvolvimento Científico, Tecnológico do Maranhão (FAPEMA), Universidade Federal do Maranhão (UFMA) e Universidade Federal do Piauí (UFPI) pela ajuda financeira.

\section{Referências}

Al-Qunaieer, F. S., Tizhoosh, H. R., and Rahnamayan, S. (2014). Multi-resolution level sets with shape priors: a validation report for $2 \mathrm{~d}$ segmentation of prostate gland in $\mathrm{t} 2 \mathrm{w}$ $\mathrm{mr}$ images. Journal of digital imaging, 27(6):833-847.

Bloch, N., Madabhushi, A., Huisman, H., et al. (2015). Nci-isbi 2013 challenge: automated segmentation of prostate structures.

Duda, R. (1973). Pattern classification and scene analysis. New-York, London, Sydny, Tronto A Wiley-Interscience Publication. 
Gonzalez, R. and Woods, R. (2008). Digital image processing. Pearson, Prentice Hall.

INCA (2018). Instituto nacional do câncer - câncer -tipo - prostata. http://www2.inca.gov.br/wps/wcm/connect/tiposdecancer/site/home/prostata. Acessado: 23 março 2018.

Karimi, D., Samei, G., Shao, Y., and Salcudean, T. (2017). A novel deep learning-based method for prostate segmentation in 2 -weighted magnetic resonance imaging.

Krizhevsky, A., Sutskever, I., and Hinton, G. E. (2012). Imagenet classification with deep convolutional neural networks. In Advances in neural information processing systems, pages 1097-1105.

LeCun, Y., Bottou, L., Bengio, Y., and Haffner, P. (1998). Gradient-based learning applied to document recognition. Proceedings of the IEEE, 86(11):2278-2324.

Li, A., Li, C., Wang, X., Eberl, S., Feng, D. D., and Fulham, M. (2013). Automated segmentation of prostate $\mathrm{mr}$ images using prior knowledge enhanced random walker. In Digital Image Computing: Techniques and Applications (DICTA), 2013 International Conference on, pages 1-7. IEEE.

Maan, B. and van der Heijden, F. (2012). Prostate mr image segmentation using 3d active appearance models. MICCAI Grand Challenge: Prostate MR Image Segmentation, 2012.

Mahapatra, D. and Buhmann, J. M. (2014). Prostate mri segmentation using learned semantic knowledge and graph cuts. IEEE Transactions on Biomedical Engineering, 61(3):756-764.

Ronneberger, O., Fischer, P., and Brox, T. (2015). U-net: Convolutional networks for biomedical image segmentation. In International Conference on Medical image computing and computer-assisted intervention, pages 234-241. Springer.

Simonyan, K. and Zisserman, A. (2014). Very deep convolutional networks for large-scale image recognition. arXiv preprint arXiv:1409.1556.

Smith, K., Clark, K., Bennett, W., Nolan, T., Kirby, J., Wolfsberger, M., Moulton, J., Vendt, B., and Freymann, J. (2015). Data from prostate-3t. http:// dx.doi.org/10.7937/K9/TCIA.2015.QJTV5IL5. Acessado: 01 agosto 2017.

Society, A. C. (2018). About prostate cancer. https://www.cancer.org/cancer/prostatecancer.html. Acessado: 23 março 2018.

Szegedy, C., Liu, W., Jia, Y., Sermanet, P., Reed, S., and Anguelov, D. (2015). \& rabinovich, a.(2015). going deeper with convolutions. In Proceedings of the IEEE conference on computer vision and pattern recognition, pages 1-9.

Tian, Z., Liu, L., Zhang, Z., and Fei, B. (2018). Psnet: prostate segmentation on mri based on a convolutional neural network. Journal of Medical Imaging, 5(2):021208.

Yang, X., Rossi, P., Ogunleye, T., Marcus, D. M., Jani, A. B., Mao, H., Curran, W. J., and Liu, T. (2014). Prostate ct segmentation method based on nonrigid registration in ultrasound-guided ct-based hdr prostate brachytherapy. Medical physics, 41(11). 
Yang, X., Zhan, S., and Xie, D. (2016). Landmark based prostate mri segmentation via improved level set method. In 2016 IEEE 13th International Conference on Signal Processing (ICSP), pages 29-34.

Zeiler, M. D. A. (2012). An adaptive learning rate method. arxiv preprint. arXiv preprint arXiv: 1212.5701 .

Zhu, Q., Du, B., Turkbey, B., Choyke, P. L., and Yan, P. (2017). Deeply-supervised cnn for prostate segmentation. In Neural Networks (IJCNN), 2017 International Joint Conference on, pages 178-184. IEEE. 